1. MBBS, M.Phil Pharmacology Assistant Professor Department of Pharmacology Mohtarma Banazir Bhutto Shaheed Medical College Mirpur.

2. MBBS, M.Phil Pharmacology

Assistant Professor

Department of Pharmacology

Rashid Latif Medical College Lahore.

3. MBBS

Medical Officer

DHQ Teaching Hospital

Sargodha Department.

4. MBBS, M.Phil Pharmacology Department of Pharmacology Sargodha Medical College Sargodha.

5. MBBS

Senior Demonstrator Department of Hematology

Rashid Latif Medical College Lahore.

Correspondence Address:

Dr. Rehana Mukhtar

Medical Officer

DHQ Teaching Hospital

Sargodha Department.

drrehanamukhtar0063@yahoo.com

Article received on:

22/08/2017

Accepted for publication:

$15 / 11 / 2017$

Received after proof reading:

$28 / 02 / 2018$

\section{VIRAL HEPATITIS;}

DIABETICS PATIENTS INFECTED WITH HEPATITIS B OR C VIRUS; METFORMIN INTAKE IS ASSOCIATED WITH DECREASED INCIDENCE OF HEPATOCELLULAR CARCINOMA

\section{Sohail Iqbal', Rao Salman Aziz ${ }^{2}$, Rehana Mukhtar ${ }^{3}$, Farwa Naqvi ${ }^{4}$, Maheen Rana}

ABSTRACT... Objectives: Diabetes mellitus has been shown to be an independent risk factor for hepatocellular carcinoma in populations at risk. Different anti-diabetic drugs have been shown to have different effects on incidence of cancers in diabetic patients. Data Source: Outpatient and inpatient hospital records as well as fresh cases. Design of Study: Hospital based retrospective case control study. Setting: DHQ, Sargodha. Period: Fifteen years (10 years retrospective, 5 years prospective, from the starting date of study). Materials \& Methods: In a hospital based study we recorded the incidence of HCC in patients otherwise considered to be at risk. We also observed whether anti-diabetic monotherapy has any effects on HCC occurrence in our target population. Results: We found out that incidence of HCC in diabetic patients with a concomitant HBV or HCV infection was at least 1.5 times higher than non-diabetic hepatitis patients. The incidence of HCC reduced in patients using metformin as compared to sulfonylureas. Conclusion: Diabetes is an independent risk factor for hepatocellular carcinoma and metformin use is correlated with reduced HCC occurrence in risk groups.

Key words: $\quad$ Diabetes mellitus, Hepatitis B or C virus, Hepatocellular Carcinoma

Article Citation: Iqbal S, Aziz RS, Mukhtar R, Naqvi F, Rana M. Viral hepatitis; Diabetics patients infected with hepatitis $B$ or $C$ virus; metformin intake is associated with decreased incidence of hepatocellular carcinoma. Professional Med J 2018;25(3):400-408. DOI:10.29309/TPMJ/18.4270

\section{INTRODUCTION}

Liver cancer is sixth most common cancer in the world. In males it is the fifth most common cancer while in females it ranks seventh. In spite of it being sixth most common cancer worldwide, liver cancer is associated with more mortalities than many other cancers. ${ }^{1}$ This is evident from the fact that it is third most common cause of cancer related deaths worldwide in both genders. Lung cancer is by far the most common cancer in world as well as most common cause of cancer related deaths followed by stomach cancer as the second most common cause of cancer related deaths in both sexes of all ages. ${ }^{1}$

According the World Health Organization's subsidiary International Agency for Research on Cancer (IARC) reports, in the year 2008, 748,000 new liver cancer cases were recorded while it was responsible for 696,000 deaths in the year 2008 alone. ${ }^{2}$ Around 85 percent of the total liver cancer cases occur in less developed countries. It is also important to note here that more than 50 percent of all liver cancer cases occur in china alone. This data points out to the gravity of situation and also the important variations on liver cancer incidence around the world. ${ }^{2}$

Hepatocellular carcinoma is the major form of liver cancer that involves the primary functional cells of liver i.e. hepatocytes. Hepatocellular carcinoma accounts for 85 - 90 percent of all liver cancer cases. ${ }^{3}$ Multiple etiological factors are responsible for hepatocellular carcinoma. Infection with two most dangerous hepatitis viruses, namely hepatitis B virus and hepatitis $C$ virus, has been recorded as the underlying etiology of more than three fourth (78\%) of total hepatocellular carcinoma cases. ${ }^{2}$ Other important factors responsible for hepatocellular carcinoma include; exposure to fungal toxin (aflatoxin), hemochromatosis, chronic alcoholism and anabolic steroids. All these factors combined account for only $22 \%$ of total HCC cases. ${ }^{2}$ 
Hepatitis B virus is the most common cause of HCC that accounts for around $53 \%$ of total HCC cases alone worldwide. However, contrary to worldwide trend, hepatitis $\mathrm{C}$ virus is more common etiology underlying $\mathrm{HCC}$ in certain areas of high HCV prevalence. These areas include Japan, Pakistan and Egypt. ${ }^{4}$ Moreover, with the introduction of a vaccine against HBV and the availability of effective therapeutic options, incidence of HBV infection is expected to decline markedly in the coming years. On the contrary, no vaccine is available as yet for $\mathrm{HCV}$ and the therapies available have been found to less effective. Therefore, it is expected that in coming years HCV will take over as the most common cause of HCC worldwide.

Diabetes mellitus is a major health concern worldwide. In United States, it is the fifth most common cause of death. ${ }^{5}$ Diabetes is a disease of glucose metabolism either related to insensitivity of insulin receptors to endogenous insulin or less than normal production of insulin by pancreatic islands of Langerhans. ${ }^{6}$ Liver plays an important role in glucose metabolism therefore various studies have found a correlation between diabetes mellitus and various liver diseases such as chronic hepatitis, cirrhosis and liver failure. ${ }^{6} \mathrm{~A}$ large number of cohort ${ }^{7,8}$ as well as case control studies ${ }^{9}$ have established a link between Diabetes Mellitus and Hepatocellular Carcinoma. Although it is mostly believed that diabetes contributes to the development of $\mathrm{HCC}$ in the presence of underlying liver disease ${ }^{10}$ but strong evidence also exists which point out towards the fact that diabetes might be an independent risk factor for HCC development. ${ }^{11}$

Metformin is biguanide derivative and is the most commonly used drug as first line treatment of type 2 diabetes. ${ }^{12}$ Primarily, metformin acts by inhibiting glucose production in liver as well as reduces resistance to insulin in peripheral tissue. Consequently, glucose uptake by skeletal tissues is increased leading to an increase in utilization of glucose and reduction of plasma insulin and glucose levels. ${ }^{13}$ Multiple cohort and case control studies have established the fact that metformin treatment is associated with reduced risk of development of multiple types of solid tumors. ${ }^{14,15}$ On the contrary, patients using sulfonylureas do not exhibit such reduced risks. ${ }^{15}$

However, the evidence related to metformin's role in reducing cancer incidence is not conclusive. A recent comprehensive meta-analysis of various random controlled trials, case studies and cohort studies found out that although the relation between metformin use and decreased incidence of cancer is supported by cohort and case control studies $((\mathrm{RR}=0.70[95 \% \mathrm{Cl}=0.67-0.73]$ and $\mathrm{OR}=0.90$ [95\% $\mathrm{Cl}=0.84-0.98]$, respectively), random controlled trials do not establish a conclusive link between metformin use and decreased incidence of hepatocellular carcinoma in diabetic patients. ${ }^{15}$

In this study, we sought to generate more reliable data in a systematic manner so that a link between metformin use and hepatocellular carcinoma incidence be established. We also sought to understand the relationship between use of sulfonylureas, another class of anti-diabetic drugs, and hepatocellular carcinoma incidence. A random controlled trial was conducted over a period of seven years.

\section{MATERIALS AND METHODS}

\section{Ethical considerations}

The study was conducted in accordance with the principles of medical research ethics set forth by the Declaration of Helsinki (2008), a set of ethical principles promulgated by the World Medical Association. All patients included in the study provided an informed consent and the patients details were kept anonymous except for the two persons directly involved in data accumulation. As per WMA principles, the patients were duly assigned code numbers and any further analysis of patient data used the assigned code numbers instead of original name or any other identifications.

\section{Study population}

This study was an observational study. The study design was that of a hospital based retrospective case control study. The study spanned over a 
period of five years during which data of patients relevant to the study were retrieved for the past ten years (from the starting date of study) from the hospital records. Also, any new cases registered during the study period were also included in the population under study. Patients reporting to the hospital for complaints of various liver related malignancies of unknown origin were included in the study. In total, data of 734 patients was included in the current study (both retrospective as well as new cases). Out of these 734 patients initially recruited for the study, a total of 680 patients were ultimately selected for final analysis. Patients were further divided into two main groups based on their diabetic status. Patients younger than 20 years of age and were not included in the study.

\section{Clinical and laboratory examination}

Only those patients were finally included in the study whose records provided a reliable data for various clinical symptoms and laboratory diagnoses. The laboratory investigations included; liver function tests and serological analysis for possible hepatitis virus infection, fasting and random blood sugar and serum HbA1c levels for diabetes. Ultrasonographic examination of liver was considered as the sufficient diagnostic parameter for any possible liver injuries (except for HCC cases). In case of HCC patients, histopathological analysis of liver biopsy samples and/or computed tomographic confirmation of cancerous lesion and serum a-fetoprotein levels were also considered before including the patient in study group. Smoking and alcohol usage was inquired by careful history taking and cross questioning by a qualified and trained nurse.

Due to the unavailability of advanced diagnostic tools in past, a positive screening test for HBV or HCV was considered as sufficient diagnostic parameter for retrospective cases. In case of fresh subjects recruited for study, a positive screening test for hepatitis $\mathrm{B}$ or $\mathrm{C}$ virus was further confirmed by presence of antibodies against HBV surface antigen (anti-HbsAg) using commercial assay (Abbott Diagnostic Division, Wiesbaden; Germany) or antibodies against HCV using a third-generation line assay (Immunogenetics, Gent, Belgium). Hepatitis infection was further confirmed by qualitative PCR. For HBV PCR, protocol and primer sequences reported by (Chung, 2004) was used. While for detection of HCV RNA in patient serums, the protocol and primer sequences reported by. ${ }^{16}$ Primer sequences are given in Table-II.

\section{Anti-diabetic drug usage}

The patient's clinical records were carefully scrutinized for usage of usage of prescription antidiabetic drugs (in diabetic group). In our study, we included only patients using a single type of antidiabetic drug for at least one year continuously. Patients using more than one drug at a time were not included in the study. We compared the risk of development of hepatocellular carcinoma in diabetic patients using metformin or sulfonylureas only. Patients using any other drug like insulin or the patients who switched between different drug combination regimens from the time of enrolment to their death were excluded from the study.

\section{Statistical Analysis}

All statistical analysis was carried out using PASW Statistics for Windows, Version 18.0. (SPSS Inc. Chicago) The patients were divided into different groups for descriptive analysis. (Metformin vs Sulfonylureas and Diabetic Vs Non-Diabetic). Comparison between groups was made by using two way ANNOVA.

\section{RESULTS}

A total of 680 (368 Non-diabetic Vs 312 Diabetic) patients matching our inclusion criteria were finally selected for this study. The demographic description patient cohort is given in Table-I. Our observation concluded HCV as the major cause of chronic liver disease as well as hepatocellular carcinoma (See Table-III). Out of the total $354 \mathrm{HBV}$ infected patients, 134 (37.9\%) patients developed HCC over the course of study. There was a total of $266 \mathrm{HCV}$ infected patients in our study, out of which 110 (41.3\%). The data indicates that HCV infection is more correlated with development of $\mathrm{HCC}$ over the course of years.

Our observations also indicated that development 
of HCC is more prevalent in patients with concomitant diabetes as compared to those without diabetes (see Figure-IV). Overall there were 274 diabetic patients with any of the viral infections. Out of these 274 diabetic patients, 131 patients developed hepatocellular carcinoma. The incidence of HCC among diabetic patients with hepatitis viral infection was $48 \%$ as compared to the non-diabetic group in which only $33 \%$ of the total patients developed HCC (113 out of 346). Our data clearly indicates that concomitant diabetes increases the risk of development of HCC in patients infected with hepatitis viruses.

The number of patients reporting for alcoholic liver damage was very low, so no reliable correlation between HCC due to alcoholism and diabetes can be made. Nonetheless, out of 7 diabetic patients reporting for the complaints of alcoholic liver damage, 2 developed HCC while in no-diabetic alcoholics, none of the 2 patients developed hepatocellular carcinoma till the time of their death.

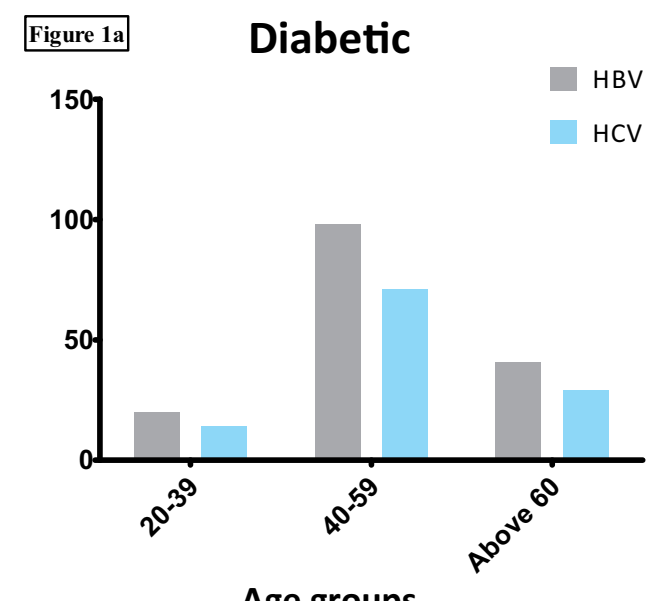

Figure-1 (a). Age distribution of hepatitis virus infection among diabetic patients

We also compared the incidence of HCC occurrence in patients using two different antidiabetic monotherapies vis-à-vis Metformin and Sulfonylureas. Among the total 312 diabetic patients, 63\% (196) were using metformin monotherapy during the course of study. While the rest of $37 \%$ (116) patients used sulfonylureas from the time of enrolment in study to their death or ending period of study, whichever occurred earlier. Our observations indicated that metformin use significantly reduced the risk of development of hepatocellular carcinoma in patients at risk otherwise. The sulfonylureas group appears to have an increased risk of HCC development, however from the current study, it cannot be concluded with authenticity whether sulfonylureas increase the risk of HCC development or not. As there was no independent control group in this study, analysis of effect of sulfonylureas uses on the risk of development of HCC in patients predisposed could not be analyzed.

Our results indicate that out of 196 patients under metformin monotherapy, only 47 patients developed hepatocellular carcinoma (24\%). However, the incidence of HCC occurrence in sulfonylureas group was much higher. 84 patients out of the total 116 in sulfonylureas groups developed HCC (72\%). There were no significant differences between two groups in their mean ages, sex composition or any other factors like possibility of exposure to dietary aflatoxin, smoking or occasional alcohol consumption (alcohol usage was only considered as the major etiology underlying chronic liver damage if the dose was $>30 \mathrm{~g}$ in males and $>20 \mathrm{~g}$ in females (Corrao, et al., 1994)).

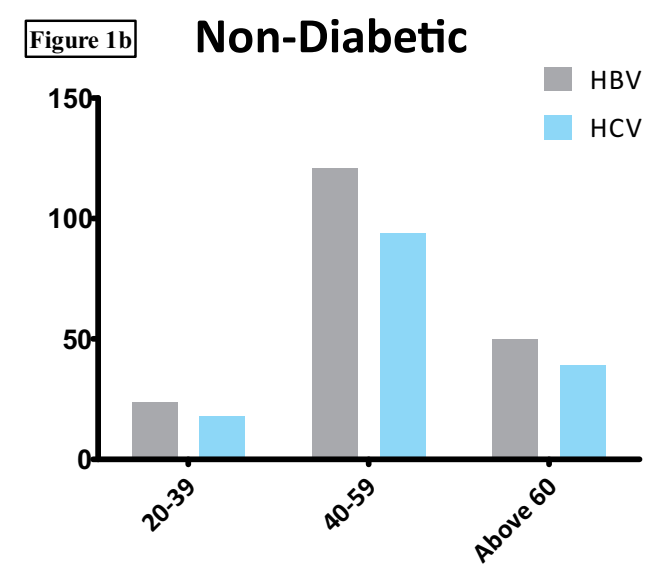

Age groups

Figure-1 (b). Represents the distribution of HBV and $\mathrm{HCV}$ infection among diabetic and non-diabetic patients in different age groups.

Figure-2 HBV infection was found to be the major underlying etiology of chronic liver disease in the patient cohort under study. HCV infection though 
found in less number of patients was correlated more with the development of HCC as compared to HBV, both in diabetic and non-diabetic groups. Figure-3. Incidence of HCC among HBV and HCV infected patients was at least 1.5 times higher in patients with concomitant diabetes than the nondiabetic group.

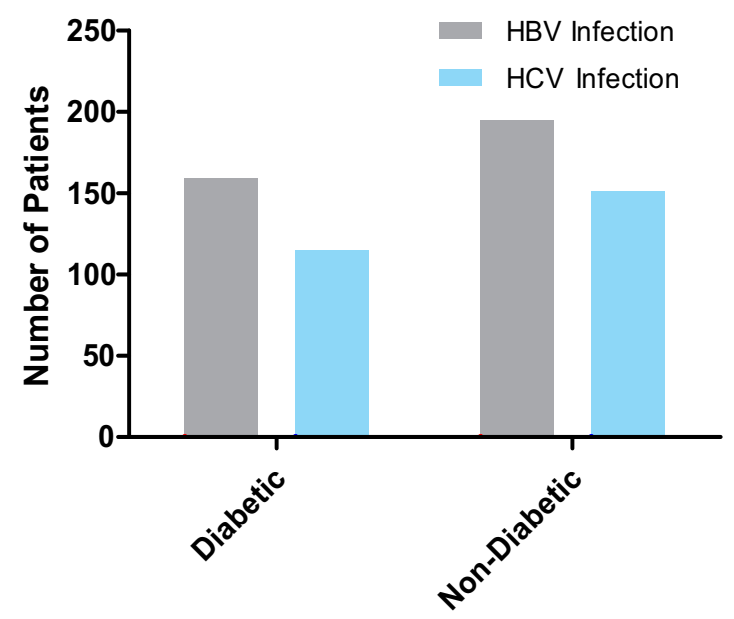

Figure-2. Total number of patients infected with a particular virus in diabetic and non-diabetic groups.

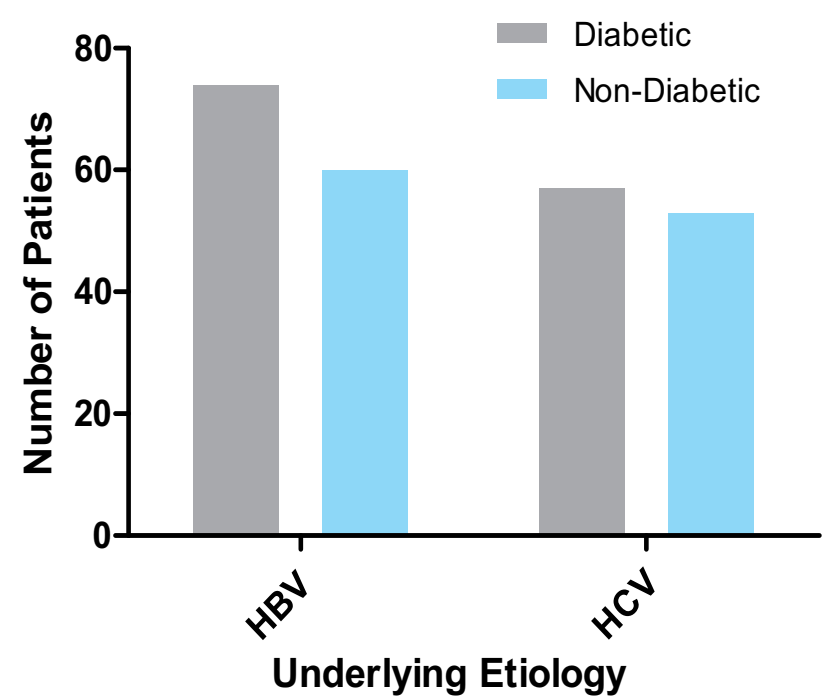

Figure-3. Incidence of Hepatocellular carcinoma in diabetic and non-diabetic patients with HBV or HCV infection

Figure-4 depicts the incidence of HCC among different drug groups. Out of total 196 patients using metformin, only 47 developed HCC. While the ratio of patients developing $\mathrm{HCC}$ was much higher in sulfonylurea group (84 out of 116 total)

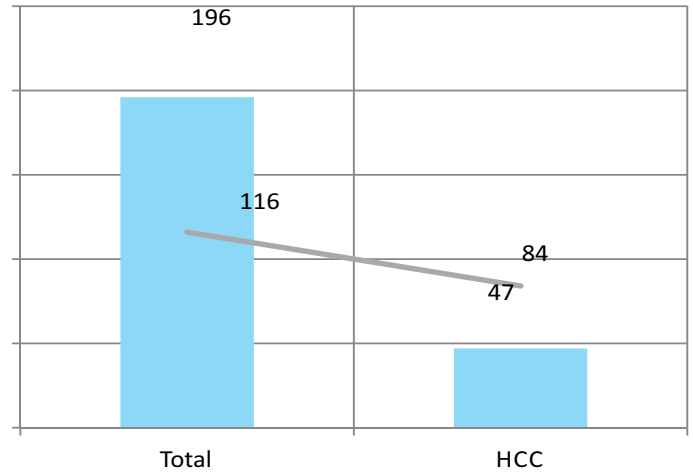

Figure-4. Incidence of HCC among patients using different anti-diabetic monotherapies

\section{DISCUSSION}

Hepatocellular carcinoma is among the major health hazards worldwide. ${ }^{17}$ According to World Health Organization reports it is the third major cause of cancer related mortality in world. ${ }^{2}$ Apart from primary etiologies like hepatitis viral infections and aflatoxin exposure, certain medical conditions have been shown to affect the hepatocarcinogenesis. Various studies have shown that diabetes, pre- or post-infection in chronic hepatitis patients increases the risk of development of HCC manifolds.

Ourstudy shows that incidence of HCC occurrence increases more than 1.5-fold in diabetic patients when compared with non-diabetic patients.

This increased incidence is independent of the hepatitis viral infection as similar trend was observed in both HBV and HCV cohorts. Therefore, diabetes can be considered as an independent risk factor in the hepatocarcinogenesis. However, the data available till date is not conclusive and a controversy exists as to what extent diabetes mellitus be considered as an independent risk factor in the development of HCC. ${ }^{18}$

In a recent comprehensive study in Taiwan, a total of 19,349 patients suffering from diabetes mellitus were followed through medical insurance claims and the records were analyzed for the development of HCC in the target group. ${ }^{19}$ When compared with a large cohort of control group (non-diabetic), it was revealed that diabetes mellitus increases the risk of HCC development at least two folds. ${ }^{19}$ 


\begin{tabular}{|c|c|c|c|c|c|}
\hline & \multicolumn{2}{|c|}{ Non-Diabetic $(n=368)$} & \multicolumn{2}{|c|}{ Diabetic $(n=312)$} & \multirow[b]{2}{*}{ p Value } \\
\hline & $\mathbf{n}$ & $\%$ & $\mathbf{n}$ & $\%$ & \\
\hline Sex & & & & & 0.94 \\
\hline Men & 202 & 54.9 & 165 & 52.9 & \\
\hline Women & 166 & 45.2 & 147 & 47.1 & \\
\hline Age (Years) & & & & & 0.96 \\
\hline $20-39$ & 45 & 12.2 & 39 & 12.5 & \\
\hline $40-59$ & 229 & 62.2 & 193 & 61.9 & \\
\hline$\geq 60$ & 94 & 25.5 & 80 & 25.6 & \\
\hline Mean age (Years \pm SD) & \multicolumn{2}{|c|}{$52.5 \pm 13.9$} & \multicolumn{2}{|c|}{$52.7 \pm 14.2$} & 0.74 \\
\hline \multicolumn{6}{|l|}{ Comorbidities } \\
\hline Cirrhosis (NBNC) & 6 & 1.6 & 10 & 3.2 & $<0.0001$ \\
\hline Alcoholic Liver Damage & 2 & 0.5 & 7 & 2.2 & $<0.0001$ \\
\hline HBV infection & 195 & 53 & 159 & 51 & $<0.0001$ \\
\hline HCV infection & 151 & 41 & 115 & 37 & $<0.0001$ \\
\hline
\end{tabular}

Table-I. Patient demographic data and details of comorbidities

\section{Forward Primer}

Reverse Primer

HBV Surface Antigen (Chung, 2004)

First Round PCR

CCTGCTGGTGGCTCGAGTTC

CAAACGGGCAACATACCTTG

Nested PCR

ACATCAGGATTCCTAGGACC

HCV 5' UTR (Ahmad, et al., 2007)

First Round PCR

GCCATGGCGTTAGTATGAGT

Nested PCR

GTGCAGCCTCCAGGACCC

CGCAGACACATCCAGCGATA

GTGCACGGTCTACGAGACCT

CCGTGAGCGTTCGTGGGATA

Table-Il. Primer sequences for viral nucleic acid detection

\begin{tabular}{|c|c|c|c|}
\hline Etiology & $\begin{array}{c}\text { Diabetic } \\
\text { N (\%) }\end{array}$ & $\begin{array}{c}\text { Non-Diabetic } \\
\text { N (\%) }\end{array}$ & Odds Ratio \\
\hline HBV & $74(46.5)$ & $60(30.8)$ & $5.34(2.44-11.67)$ \\
\hline $\mathrm{HCV}$ & 57 (49.6) & $53(35.1)$ & $10.12(2.19-46.80)$ \\
\hline Alcoholism & 2 & Nil & 1.00 \\
\hline
\end{tabular}

A similar large scale prospective study in Singapore 20 found out similar results. Hepatitis viral infections account for more than two thirds of all HCC case worldwide, however the study further established that diabetes mellitus can be an independent risk factor in the development of HCC even in the absence of serological markers for hepatitis viral infection. The study pinpoints the risk associated with diabetes mellitus. Several other studies in various different geographical locations have found a similar correlation. For example ${ }^{21}$ and ${ }^{22}$ in United States, ${ }^{23}$ and ${ }^{24}$ in Japan. These multiple studies have established beyond doubt that diabetes mellitus is correlated with the incidence of HCC. But this risk of HCC development is not restricted to groups already at risk of HCC development. Although studies have shown that occurrence of diabetes mellitus increases the risk of $\mathrm{HCC}$ in $\mathrm{HBV}$ and/or HCV patients, diabetes is also an independent risk factor for HCC.

The mechanism by which metformin helps reduce cancer risk is still not fully understood, however studies have proposed that inhibition of hypeinsulinemia might be indirectly involved in reduced risk of cancer in metformin treated patients. ${ }^{25}$ Many studies in multiple types of 
cancers have established the usefulness of metformin in reducing risk of cancer. For example, in one large populations based study conducted in Netherlands which included around 2.5 million people, it was found that metformin usage reduced the cumulative risk for development of cancer as compared to the risk faced by patients using other drug regimens $>{ }^{26}$ In another study on 973 pancreatic adenocarcinoma patients found out that the risk of development of cancer in metformin using diabetic patients was much less than the controls (O.R 0.38).

Studies have also revealed microRNA mediated mechanisms to be involved in metformin induced arrest in cancer cell growth. It has been found that metformin modulates the expression of various microRNAs including mir192 and mir26a. Mir26a has been found to inhibit cell proliferation and caused increased apoptosis. ${ }^{27}$

In our study we also found that use of sulfonylurea derivatives does not affect the incidence of cancer. Data from a few reports raises the suspicion that use of sulfonylureas might contribute to the increased risk of cancer development. ${ }^{28}$ However our results disagree with the conclusions drawn earlier. Further studies might be needed to establish conclusive evidence. Our results are in agreement with previous studies and emphasize the need for the measures to be taken in diabetic patients who are also at the risk of HCC development due to other underlying etiologies.

\section{CONCLUSION}

Diabetes is an independent risk factor for hepatocellular carcinoma and metformin use is correlated with reduced HCC occurrence in risk groups

\section{Copyright@ 15 Nov, 2017.}

\section{REFERENCES}

1. GLOBOCAN 2012 v1.0, Cancer Incidence and Mortality Worldwide: IARC CancerBase No. 11 [Internet]. International Agency for Research on Cancer. 2013 [cited 11/03/2014]. Available from: http:// globocan.iarc.fr.

2. Jemal A, Center MM, DeSantis C, Ward EM. Global patterns of cancer incidence and mortality rates and trends. Cancer Epidemiology and Prevention Biomarkers. 2010;19(8):1893-907.

3. Farazi PA, DePinho RA. Hepatocellular carcinoma pathogenesis: from genes to environment. Nature Reviews Cancer. 2006;6(9):674-87.

4. Umer $M$, Iqbal $M$. Hepatitis $\mathbf{C}$ virus prevalence and genotype distribution in Pakistan: Comprehensive review of recent data. World Journal of Gastroenterology. 2016;22(4):1684-700.

5. Harris MI, Flegal KM, Cowie CC, Eberhardt MS, Goldstein DE, Little RR, et al. Prevalence of diabetes, impaired fasting glucose, and impaired glucose tolerance in US adults: the Third National Health and Nutrition Examination Survey, 1988-1994. Diabetes Care. 1998;21(4):518-24.

6. Hassan MM, Curley SA, Li D, Kaseb A, Davila M, Abdalla $E K$, et al. Association of diabetes duration and diabetes treatment with the risk of hepatocellular carcinoma. Cancer. 2010;116(8):1938-46.

7. Chen CL, Yang HI, Yang WS, Liu CJ, Chen PJ, You SL, et al. Metabolic factors and risk of hepatocellular carcinoma by chronic hepatitis $B / C$ infection: a follow-up study in Taiwan. Gastroenterology. 2008; 135(1):111-21.

8. Wideroff L, Gridley G, Chow W-H, Linet M, Mellemkjaer $\mathrm{L}$, Olsen $\mathrm{JH}$, et al. Cancer incidence in a populationbased cohort of patients hospitalized with diabetes mellitus in Denmark. Journal of the National Cancer Institute. 1997; 89(18):1360-5.

9. Davila J, Morgan R, Shaib Y, McGlynn K, El-Serag $H$. Diabetes increases the risk of hepatocellular carcinoma in the United States: a population based case control study. Gut. 2005;54(4):533-9.

10. Veldt BJ, Chen W, Heathcote EJ, Wedemeyer H, Reichen J, Hofmann WP, et al. Increased risk of hepatocellular carcinoma among patients with hepatitis $\mathbf{C}$ cirrhosis and diabetes mellitus. Hepatology. 2008;47(6):185662.

11. El-Serag HB, Hampel $H$, Javadi F. The association between diabetes and hepatocellular carcinoma: a systematic review of epidemiologic evidence. Clinical Gastroenterology and Hepatology. 2006; 4(3):369-80.

12. Kirpichnikov D, McFarlane SI, Sowers JR. Metformin: an update. Annals of Internal Medicine. 2002; 137(1):2533.

13. Bailey CJ, Turner RC. Metformin. New England Journal of Medicine. 1996;334(9):574-9.

14. DeCensi A, Puntoni M, Goodwin P, Cazzaniga M, 
Gennari A, Bonanni B, et al. Metformin and cancer risk in diabetic patients: a systematic review and meta-analysis. Cancer Prevention Research. 2010;3(11):1451-61.

15. Thakkar B, Aronis KN, Vamvini MT, Shields K, Mantzoros CS. Metformin and sulfonylureas in relation to cancer risk in type II diabetes patients: a meta-analysis using primary data of published studies. Metabolism. 2013;62(7):922-34.

16. Ahmad N, Asgher M, Shafique M, Qureshi JA. An evidence of high prevalence of Hepatitis $C$ virus in Faisalabad, Pakistan. Saudi Medical Journal. 2007;28(3):390-5.

17. Umer M, Qureshi SA, Hashmi ZY, Raza A, Ahmad J, Rahman $M$, et al. Promoter hypermethylation of Wnt pathway inhibitors in hepatitis $C$ virus-induced multistep hepatocarcinogenesis. Virology Journal. 2014;11(1):117-26.

18. Gao C, Yao S-K. Diabetes mellitus: a "true" independent risk factor for hepatocellular carcinoma. Hepatobiliary \& Pancreatic Diseases International. 2009;8(5):465-73.

19. Lai S-W, Chen P-C, Liao K-F, Muo C-H, Lin C-C, Sung F-C. Risk of hepatocellular carcinoma in diabetic patients and risk reduction associated with anti-diabetic therapy: a population-based cohort study. The American Journal of Gastroenterology. 2012;107(1):4652.

20. Koh W, Wang R, Jin A, Yu M, Yuan J. Diabetes mellitus and risk of hepatocellular carcinoma: findings from the Singapore Chinese Health Study. British Journal of Cancer. 2013;108(5):1182-8.

21. Coughlin SS, Calle EE, Teras LR, Petrelli J, Thun MJ.
Diabetes mellitus as a predictor of cancer mortality in a large cohort of US adults. American Journal of Epidemiology. 2004;159(12):1160-7.

22. El-serag HB, Tran T, Everhart JE. Diabetes increases the risk of chronic liver disease and hepatocellular carcinoma. Gastroenterology. 2004;126(2):460-8.

23. Fujino Y, Mizoue T, Tokui N, Yoshimura T. Prospective study of diabetes mellitus and liver cancer in Japan. Diabetes/Metabolism Research and Reviews. 2001;17(5):374-9.

24. Inoue M, Iwasaki M, Otani T, Sasazuki S, Noda M, Tsugane S. Diabetes mellitus and the risk of cancer: results from a large-scale population-based cohort study in Japan. Archives of Internal Medicine. 2006;166(17):1871-7.

25. Noto H, Goto A, Tsujimoto T, Noda M. Cancer risk in diabetic patients treated with metformin: a systematic review and meta-analysis. PloS One. 2012;7(3):e33411.

26. Ruiter R, Visser LE, van Herk-Sukel MP, Coebergh J-WW, Haak HR, Geelhoed-Duijvestijn PH, et al. Lower risk of cancer in patients on metformin in comparison with those on sulfonylurea derivatives. Diabetes Care. 2012;35(1):119-24.

27. Del Barco S, Vazquez-Martin A, Cufí S, OliverasFerraros C, Bosch-Barrera J, Joven J, et al. Metformin: multi-faceted protection against cancer. Oncotarget. 2011;2(12):896-917.

28. Bowker SL, Majumdar SR, Veugelers P, Johnson JA. Increased cancer-related mortality for patients with type 2 diabetes who use sulfonylureas or insulin. Diabetes Care. 2006;29(2):254-8. 
You can easily judge the character of a man by how he treats those who can do nothing for him.

\section{- James D. Miles -}

\begin{tabular}{|c|l|l|l|}
\hline \multicolumn{3}{|c}{ AUTHORSHIP AND CONTRIBUTION DECLARATION } \\
\hline Sr. \# & \multicolumn{1}{|c|}{ Author-s Full Name } & \multicolumn{1}{|c|}{ Contribution to the paper } & Author=s Signature \\
\hline 1 & Sohail lqbal & Performed experiments \\
\hline 2 & Rao Salman Aziz & $\begin{array}{l}\text { Literature, Introduction and } \\
\text { methodology } \\
\text { Analyzed data }\end{array}$ \\
\hline 4 & Rehana Mukhtar & Farwa Naqvi & Performed experiments \\
\hline 5 & Maheen Rana & $\begin{array}{l}\text { Grammer mistakes, Analyze } \\
\text { results }\end{array}$ \\
\hline
\end{tabular}

